\title{
Role of Pterygopalatine Fossa Block in achieving Relatively Bloodless Field during Endoscopic Sinus Surgery
}

\author{
${ }^{1}$ Reji Mathew, ${ }^{2}$ Chandrakala Srinivasa, ${ }^{3}$ Vinayababu Sathyanarayana, ${ }^{4}$ Sindhura Suryanarayana, ${ }^{5}$ Pooja Harsha
}

\begin{abstract}
Aim: To study the efficacy of pterygopalatine fossa (PPF) block with $2 \%$ xylocaine and 1:80,000 adrenaline on bleeding during endoscopic sinus surgery (ESS).

Materials and methods: Single-blinded prospective comparative study was conducted on patients who underwent bilateral functional endoscopic sinus surgery after obtaining written informed consent. The effect of PPF infiltration was tested by injecting $2 \%$ xylocaine with adrenaline on test side, and saline on the control side. The test and the control side were determined by block randomization technique. The surgeon was blinded as to which side the test and control drug was to be given. The surgical field was assessed and graded every 15 minutes on both sides according to Boezaart and Vander Merwe scoring system, and the results were compared and analyzed using Mann-Whitney $U$ test.
\end{abstract}

Results: Thirty-two patients were included in the study, of whom $59.4 \%$ were males. Ethmoidal polyposis was the most common diagnosis (50\%). The median computed tomography grade and the endoscopic grade of disease in test side and control side were comparable. The mean blood loss grade on test side was $9.0 \pm 3.5$ and in control side was $10.9 \pm 3.5$, and the difference was statistically significant. Significant positive correlation was observed between preoperative endoscopic grade of the disease and blood loss during surgery. A statistically significant correlation was also observed between the vital parameters and blood loss.

Conclusion: Pterygopalatine fossa infiltration with $2 \%$ xylocaine and 1:80,000 adrenaline reduces bleeding during ESS.

Clinical significance: This study is pivotal in devising an additional method for providing a clear bloodless operative field which to date is one of the major challenges faced by the otorhinolaryngologist.

Keywords: Control of bleeding, Endoscopic sinus surgery, Prospective case-control study, Pterygopalatine fossa.

How to cite this article: Mathew R, Srinivasa C, Sathyanarayana V, Suryanarayana S, Harsha P. Role of Pterygopalatine

\footnotetext{
${ }^{1,5}$ Postgraduate Student, ${ }^{2,3}$ Associate Professor, ${ }^{4}$ Assistant Professor

${ }^{1-5}$ Department of Otorhinolaryngology-Head and Neck Surgery, Sri Devaraj Urs Medical College, Kolar, Karnataka India

Corresponding Author: Chandrakala Srinivasa, Associate Professor, Department of Otorhinolaryngology-Head and Neck Surgery, Sri Devaraj Urs Medical College, Kolar, Karnataka India, Phone: +919481451264, e-mail: chanduharish24@yahoo.com
}

Fossa Block in achieving Relatively Bloodless Field during Endoscopic Sinus Surgery. Clin Rhinol An Int J 2017;10(2):62-65.

Source of support: Nil

Conflict of interest: None

\section{INTRODUCTION}

Functional endoscopic sinus surgery (FESS) is the most physiological and well-accepted treatment for chronic sinus disease that is unresponsive to medical treatment. ${ }^{1}$ In spite of recent advances in technology, controlling the bleeding during endoscopic sinus surgery (ESS) remains a challenge.

The surgical field in ESS is very narrow and is surrounded by vital structures, such as orbit and brain. Even a small amount of bleed during surgery can soil the tip of the endoscope and obscure the field. Repeated soiling of the tip of the endoscope prolongs the procedure. Operating under such compromised conditions in addition to the narrow and delicate field increases the risk of injury to the adjacent vital structures. Also, if performed under poor visualization, damage to the mucosa may further lead to postoperative synechiae formation. Hence, an optimal bloodless field is crucial for the surgeon. ${ }^{2}$

Purpose of this study is to devise a method of obtaining bloodless field for the performing surgeon.

Various techniques have been used to secure a dry operating field, which includes Fowler's position, hypotensive agents like beta-adrenergic blockade, and preoperative steroids and topical vasoconstrictors. However, with all these techniques, excessive bleeding still remains a problem during ESS. 3,4

As the maxillary artery is the major source of supply to the nose, vasoconstriction of this artery may significantly reduce the blood supply to the nose, thus minimizing bleeding during the procedure. ${ }^{2}$ This artery can be accessed via greater palatine foramen.

\section{MATERIALS AND METHODS}

The study was conducted at a tertiary care hospital after approval by institutional ethical committee.

A detailed clinical history was taken, and complete ear, nose, and throat examination was performed on all 
the patients. Patients were subjected to diagnostic nasal endoscopy and computed tomography (CT) imaging, and the disease was graded using Lund and Mackay system. Those subjects with bilateral similar grading were included in the study.

\section{Inclusion Criteria}

All patients above the age of 18 years with chronic rhinosinusitis or polyposis who are undergoing ESS were included.

\section{Exclusion Criteria}

- Asymmetrical disease

- Poorly controlled hypertension

- Bleeding disorders

- Use of anticoagulants

- Acute infections

Written informed consent was obtained from all the patients before enrolling them to the study. A total of 32 patients with bilateral disease were enrolled for the study. All the patients were treated preoperatively with topical steroids and an antibiotic course. All relevant investigations required for preanesthetic evaluation were done, and patients were prepared for surgery.

Since all the patients had bilateral disease, one nostril was used as test side and the other side acted as control. The test and the control side for each patient were determined by block randomization technique. Surgeon was given two similar syringes marked left and right filled with either drug or saline for infiltration. Surgeon carried out the procedure on all patients who were blinded to the side of test and control. In addition to the pterygopalatine fossa (PPF) block, both the nostrils were infiltrated with $2 \mathrm{~mL}$ of $2 \%$ xylocaine with 1:200,000 adrenaline at the beginning of the surgery. Infiltration was done at the axilla of the middle turbinate, along the anterior insertion of the uncinate process and head of the middle turbinate. The surgical procedure was alternated between both the sides every 30 minutes and bleeding was assessed using Boezaart and Vander Merwe scale every 15 minutes. The subjects underwent clearance of disease from all the sinuses on both sides.

\section{Infiltration Technique}

The greater palatine foramen is located over the hard palate just anterior to the posterior edge of the hard palate opposite the second molar tooth, halfway between the tooth and the midline of the hard palate. This point is palpated and felt as a depression and that point is marked and identified on monitor. The needle used for infiltration was bent at $25 \mathrm{~mm}$ from the tip at an angle of $45^{\circ}$ in order to perform an effective infiltration of the PPF. After aspirating to ensure that the needle was not in a blood vessel, the PPF was infiltrated with the test drug ( $2 \%$ xylocaine and 1:80,000 adrenaline) on one side. Same procedure was followed on the other side and saline was infiltrated.

\section{Assessment of Bleeding}

Surgical field was assessed by the operating surgeon for bleeding every 15 minutes on both test and control side, using Boezaart and Vander Merwe scale (Table 1). The total of all the grades was taken and compared with the control side (Table 2). The vital parameters like mean arterial pressure (MAP), heart rate (HR), blood pressure (BP), and residual carbon dioxide were documented throughout the procedure.

\section{Statistical Analysis}

Data were entered into Microsoft Excel data sheet and were analyzed using Statistical Package for the Social Sciences version 22 software. Categorical data were represented in the form of frequencies and proportions. Chi-square was used as test of significance. Continuous data were represented as mean and standard deviation. Independent t-test or Mann-Whitney $U$ test was used as test of significance to identify the mean difference between two groups; $\mathrm{p}<0.05$ was considered as statistically significant.

\section{RESULTS}

A total of 32 patients with bilateral sinonasal disease were enlisted for the study. The mean age of the subjects was $39.1 \pm 15.2$ years. Majority of the patients were males $(59.4 \%)$.

Most common diagnosis in the subjects was bilateral ethmoidal polyposis (56.25\%) followed by chronic rhinosinusitis (37.5\%). Allergic fungal sinusitis with polyposis was found in two patients $(6.25 \%)$. Mean duration of the illness was 1.47 years.

Table 1: Boezaart and Vander Merwe grading

\begin{tabular}{ll}
\hline Grades & Endoscopic grading of nasal bleeding \\
\hline 0 & No bleeding (cadaveric conditions) \\
I & Slight bleeding - no suctioning required \\
II & $\begin{array}{l}\text { Slight bleeding - occasional suctioning required } \\
\text { III }\end{array}$ \\
& $\begin{array}{l}\text { Slight bleeding - frequent suctioning required; bleeding } \\
\text { threatens surgical field a few seconds after }\end{array}$ \\
IV & $\begin{array}{l}\text { Moderate bleeding - frequent suctioning required and } \\
\text { bleeding threatens surgical field directly after }\end{array}$ \\
V & $\begin{array}{l}\text { Severe bleeding - constant suctioning required; bleeding } \\
\text { appears faster than it can be removed by suction; surgical } \\
\text { filed severely threatened and surgery usually is not } \\
\text { possible }\end{array}$ \\
\end{tabular}


Table 2: Boezaart scoring of subjects on test and control side

\begin{tabular}{|c|c|c|}
\hline \multirow[b]{2}{*}{ Patients } & \multicolumn{2}{|c|}{ Surgical grade } \\
\hline & Test side & Control side \\
\hline 1 & 13 & 16 \\
\hline 2 & 6 & 10 \\
\hline 3 & 10 & 10 \\
\hline 4 & 7 & 7 \\
\hline 5 & 8 & 16 \\
\hline 6 & 11 & 15 \\
\hline 7 & 4 & 6 \\
\hline 8 & 10 & 10 \\
\hline 9 & 10 & 12 \\
\hline 10 & 12 & 15 \\
\hline 11 & 6 & 10 \\
\hline 12 & 11 & 16 \\
\hline 13 & 8 & 11 \\
\hline 14 & 19 & 19 \\
\hline 15 & 6 & 7 \\
\hline 16 & 6 & 13 \\
\hline 17 & 13 & 6 \\
\hline 18 & 5 & 5 \\
\hline 19 & 16 & 14 \\
\hline 20 & 6 & 10 \\
\hline 21 & 12 & 12 \\
\hline 22 & 6 & 11 \\
\hline 23 & 4 & 6 \\
\hline 24 & 8 & 8 \\
\hline 25 & 12 & 12 \\
\hline 26 & 7 & 13 \\
\hline 27 & 7 & 11 \\
\hline 28 & 6 & 8 \\
\hline 29 & 8 & 12 \\
\hline 30 & 12 & 9 \\
\hline 31 & 10 & 9 \\
\hline 32 & 9 & 9 \\
\hline
\end{tabular}

The preoperative mean endoscopic grading on test and control side was 2, and CT grading was 4 on the test side and 5 on control side. The endoscopic and CT grading was matched on both the sides so that results could be compared.

Left nostril was the test side in 12 patients and right in the remaining 20 patients. The mean surgical grade on the test side was $9.0 \pm 3.5$ and on the control side was $10.9 \pm$ 3.5. The difference in mean surgical grade was statistically significant $(p=0.034)$ (Table 3). A correlation between preoperative endoscopic and $\mathrm{CT}$ grade and intraoperative

Table 3: Comparison of surgical grade between test and control side

\begin{tabular}{lllllll}
\hline & \multicolumn{3}{l}{ Injection site } & & \multicolumn{3}{c}{ Control site } & \\
\cline { 2 - 3 } & Mean & $S D$ & & Mean & $S D$ & p-value \\
\hline Endoscopic grade & 1.9 & 1.0 & & 1.8 & 1.0 & 0.02 \\
CT grade & 4.5 & 1.3 & & 4.7 & 1.5 & 0.347 \\
Blood loss & 9.0 & 3.5 & & 10.9 & 3.5 & 0.034 \\
\hline
\end{tabular}

SD: Standard deviation
Table 4: Correlation between surgical grade and endoscopic/ CT and vital parameters

\begin{tabular}{lll}
\hline & Correlation coefficient & $p$-value \\
\hline Endoscopic grading & 0.041 & 0.02 \\
CT scan grade & 0.172 & 0.347 \\
Heart rate & 0.059 & 0.001 \\
Systolic BP & 0.399 & 0.024 \\
Diastolic BP & 0.053 & 0.047. \\
Mean arterial pressure & 0.530 & 0.002 \\
\hline
\end{tabular}

surgical grade was established. Statistically significant positive correlation was found between endoscopic grade and surgical grade $(\mathrm{p}=0.007)$. A positive correlation was found between CT grade and surgical grade, but was not statistically significant $(\mathrm{p}=0.464)($ Table 4$)$.

The vital parameters like HR, BP, and MAP were also found to influence the bleeding. There was a positive correlation between these factors and the surgical grade during the procedure.

\section{DISCUSSION}

The methods used for achieving a bloodless field for performing safe and clean ESS cannot be overemphasized. There are many methods, both preoperatively and intraoperatively, to control bleeding during FESS described in the literature. The preoperative measures include the use of preoperative corticosteroids, preoperative use of $\beta$-blockers, antihypertensive agents, and surgical pledgets with oxymetazoline, phenylephrine, or cocaine, for bleeding control. The intraoperative technique includes both esthetic and surgical methods like controlled hypotension, patient positioning, use of bipolar cautery, intermittent positive pressure ventilation, warm saline irrigation, hemostatic biomaterials, topical vasoconstrictors, topical infiltration of local anesthetic and adrenaline, and the use of laryngeal mask airway. ${ }^{5-8}$ With all these techniques, obtaining a bloodless field during ESS still remains a challenge.

Pterygopalatine fossa infiltration has been used to control bleeding by many endoscopic sinus surgeons, but there is no universal consensus regarding its efficacy in achieving a bloodless field. There are studies which claim definite decrease in bleeding adopting these techniques as well as those which confront the efficacy of the technique. ${ }^{2}$

The current study was done on 32 patients with chronic rhinosinusitis and ethmoidal polyposis. The mean surgical grade on test side was $9.0 \pm 3.5$ and in control side was $10.9 \pm 3.5$. This difference in mean surgical grade was statistically significant $(\mathrm{p}=0.034)$. Two other studies reflected similar results. ${ }^{2,9}$ The current study supports their observation. In another study conducted by Valdes et $\mathrm{al}_{1}^{10}$ they found no statistical difference between injected and noninjected site in terms of surgical 
grade and blood loss. Furthermore, there was no correlation between surgical field improvement, MAP, and HR either. The effect is thought to be due to vasoconstriction effect of adrenaline on maxillary artery situated in the PPF that reduces the mucosal blood flow to the sinuses and turbinates.

The vital parameters like MAP, HR, and BP were documented throughout the procedure. It was observed that these parameters had an independent effect over bleeding. There was a significant positive correlation with vital parameters and bleeding. Another study concluded that MAP had influence on bleeding, and good operating field was achieved by maintaining MAP in the range of 65 to $78 \mathrm{~mm} \mathrm{Hg} .{ }^{11}$ In our study, the surgical field on the test side was reasonably bloodless though MAP was at a higher range $(89.3 \pm 6.4)$, and this effect could be attributed to PPF infiltration.

Other significant observation of the study is that with increasing preoperative endoscopic grade, there was a concurrent increase in bleeding at the time of surgery $(\mathrm{p}=0.007)$ (Table 2).

Although many complications have been described in literature, we did not encounter any complication related to the infiltration technique in the study subjects. This technique is relatively safe, simple with minimal complications, and also reduces bleeding significantly. Hence, it can be routinely incorporated in all ESS.

\section{CONCLUSION}

The result of this study shows that infiltration of PPF with $2 \%$ xylocaine with 1:80,000 adrenaline significantly reduces the bleeding, thus improving visualization of the surgical field during FESS. Since the technique is simple with minimal complications, it can be routinely incorporated in all ESS.

\section{CLINICAL SIGNIFICANCE}

This study is pivotal in devising an additional method for providing a clear bloodless operative field which, to date, is one of the major challenges faced by the otorhinolaryngologist.

\section{REFERENCES}

1. Chopra H, Khurana AS, Munjal M, Due K. Role of FESS in chronic sinusitis. Indian J Otolaryngol Head Neck Surg 2006 Apr;58(2):137-140.

2. Wormald PJ, Athanasiadis T, Rees G, Robinson S. An evaluation of effect of pterygopalatine fossa injection with local anesthetic and adrenalin in the control of nasal bleeding during endoscopic sinus surgery. Am J Rhinol 2005 May;19(3): 288-292.

3. Wormald, PJ. Endoscopic sinus surgery: anatomy, threedimensional reconstruction, and surgical technique. New York: Thieme; 2005. p. 7-12.

4. Drozdowski A, Sieśkiewicz A, Siemiątkowski A. Reduction of intraoperative bleeding during functional endoscopic sinus surgery. Anaesthesiol Intens Ther 2011 Jan-Mar;43(1): 45-50.

5. Thongrong C, Kasemsiri P, Carrau RL, Bergese SD. Control of bleeding in endoscopic skull base surgery: current concepts to improve hemostasis. ISRN Surg 2013 Jun;2013.

6. Ko MT, Chuang KC, Su CY. Multiple analyses of factors related to intraoperative blood loss and the role of reverse Trendelenburg position in endoscopic sinus surgery. Laryngoscope 2008 Sep;118(9):1687-1691.

7. KubitzJC, Kemming GI, Schultheiss G, Starke J, Podtschaske A, Goetz AE, Reuter DA. The influence of PEEP and tidal volume on central blood volume. Eur J Anaesthesiol 2006 Nov;23(11):954-961.

8. Kassam A, Snyderman CH, Carrau RL, Gardner P, Mintz A. Endoneurosurgical hemostasis techniques: lessons learned from 400 cases. Neurosurg Focus 2005 Jul;19(1):1-6.

9. Ismail SA, Anwar HMF. Bilateral sphenopalatine ganglion block in functional endoscopic sinus surgery under general anaesthesia. Alex J Anaesth Intensive Care 2005 Dec;8(4): 45-53.

10. Valdes CJ, Al Badaai Y, Bogado M, Samaha M. Does pterygopalatine canal injection with local anaesthetic and adrenaline decrease bleeding during functional endoscopic sinus surgery?. J Laryngol Otol 2014 Sep;128(9):814-817.

11. Sieśkiewicz A, Drozdowski A, Rogowski M. The assessment of correlation between mean arterial pressure and intraoperative bleeding during endoscopic sinus surgery in patients with low heart rate. Otolaryngol Pol 2010 Jul-Aug;64(4): 225-228. 